\title{
Neurosurgical Flexible Probe Microscopy With Enhanced Architectural and Cytological Detail
}

Hany Osman, MD, ${ }^{\mathrm{a}}$ Deena Elsahy, MD, ${ }^{\mathrm{b}}$ Veronika Slivova, $\mathrm{MS},{ }^{\mathrm{c}}$ Corey Thompson, BS, ${ }^{\mathrm{c}} \mathrm{Joseph}$ Georges, DO, PhD, ${ }^{\mathrm{d}}$ Steven Yocom, DO, ${ }^{\mathrm{d}}$ and Aaron A. Cohen-Gadol, MD, MSc, MBA ${ }^{\mathrm{e}}$

${ }^{a}$ Wellman Center for Photomedicine, Massachusetts General Hospital and Harvard Medical School, Boston, Massachusetts

${ }^{\mathrm{b}}$ Department of Obstetrics and Gynecology, Indiana University School of Medicine, Indianapolis, Indiana

${ }^{c}$ Enterprise Clinical Research Operations Biorepository, Indiana University Health, Indianapolis, Indiana

${ }^{\mathrm{d}}$ Department of Neurosurgery, Philadelphia College of Osteopathic Medicine, Philadelphia, Pennsylvania

${ }^{\mathrm{e}}$ Goodman Campbell Brain and Spine and Indiana University Department of Neurological Surgery, Indianapolis, Indiana

\section{E-mail addresses}

Hany Osman: haosman@mgh.harvard.edu

Deena Elsahy: daelsahy@iupui.edu

Veronicka Slivova: VSlivova@IUHealth.org

Corey Thompson: cthompson14@iuhealth.org

Joseph Georges: joseph.georges@asu.edu

Steven Yocom: Yocom-Steven@ cooperhealth.edu

Aaron A. Cohen-Gadol: acohenmd@gmail.com

Correspondence: Aaron Cohen-Gadol, MD, MSc, MBA, Goodman Campbell Brain and Spine, Indiana University Department of Neurological Surgery, 355 W. 16th Street, Suite 5100, Indianapolis, IN 46202. Phone: 317-362-8760; Fax: 317-924-8472; Email: acohenmd@gmail.com

Keywords: in vivo; microscopy; neurosurgery; glioma; oncology; ex vivo

Short title: Flexible fiberoptic microscopy of brain tumors

Conflict of Interest: None.

Disclosure of Funding: None.

This is the author's manuscript of the article published in final edited form as:

Osman, H., Elsahy, D., Slivova, V., Thompson, C., Georges, J., Yocom, S., \& Cohen-Gadol, A. A. (2019). Neurosurgical Flexible Probe Microscopy with Enhanced Architectural and Cytological Detail. World Neurosurgery. https://doi.org/10.1016/j.wneu.2019.05.039 


\section{Abstract}

2 Background: Microscopic delineation and clearance of tumor cells at neurosurgical excision margins potentially reduce tumor recurrence and increase patient survival. Probe-based in vivo fluorescence microscopy technologies are promising for neurosurgical in vivo microscopy.

Objective: We sought to demonstrate a flexible fiberoptic epifluorescence microscope capable of enhanced architectural and cytological imaging for in vivo microscopy during neurosurgical procedures.

Methods: Eighteen specimens were procured from neurosurgical procedures. These specimens were stained with acridine orange and imaged with a 3D-printed epifluorescent microscope that incorporates a flexible fiberoptic probe. Still images and video sequence frames were processed using frame alignment, signal projection, and pseudo-coloring, resulting in resolution enhancement and an increased field of view.

Results: Images produced displayed good nuclear contrast and architectural detail. Grade 1 meningiomas demonstrated 3D chords and whorls. Low-grade meningothelial nuclei showed streaming and displayed regularity in size, shape, and distribution. Oligodendrogliomas showed regular round nuclei and a variably staining background. Glioblastomas showed high degrees of nuclear pleomorphism and disarray. Mitoses, vascular proliferation, and necrosis were evident.

Conclusions: We demonstrate the utility of a 3D-printed, flexible probe microscope for highresolution microscopic imaging with increased architectural detail. Enhanced in vivo imaging using this device may improve our ability to detect and decrease microscopic tumor burden at excision margins during neurosurgical procedures. 


\section{Introduction}

Neurosurgical in vivo microscopy is an emerging technology that may advance our ability to detect microscopic residual tumor at tumor margins. High-grade glioma is one of the most aggressive malignancies, and it carries a grim prognosis despite maximal treatment and advances over the past decades. Their unfavorable prognosis is attributable to inevitable tumor recurrence after surgery, which leads to poor long-term patient survival. ${ }^{1,2}$ With surgery being the first line of treatment, the value of cytoreductive surgery is well documented. ${ }^{3}$ The addition of gross fluorescence guidance has further enhanced the effectiveness of tumor resection. ${ }^{4-9}$

Fluorescence-based in vivo microscopy techniques are among the most investigated techniques for in vivo neurosurgical imaging. These methods include confocal, flexible probe-based imaging and rigid miniaturized probe imaging. ${ }^{10}$ In general, flexible imaging techniques offer a practical method for imaging; however, they are limited in imaging resolution and field of view. ${ }^{10} \mathrm{~A}$ flexible probe imaging device without these limitations is therefore expected to provide a superior method for in vivo microscopy. In the current ex vivo study, we demonstrate an enhanced imaging process to produce high-resolution large-area images that resemble standard histology. We therefore aim to set the grounds for further in vivo imaging studies using this advanced technique.

\section{Materials and Methods}

\section{Specimens}

Tissues were collected during neurosurgical tumor resections. Eighteen patients were included in this study (Table 1). Patients were selected, consent was obtained, and specimens were collected according to a protocol approved by our institutional review board.

\section{Staining}

Each specimen was stained by adding approximately $50 \mu 1$ of $2 \%$ acridine-orange (SigmaAldrich, St. Louis, MO) solution to the tissues for 1 minute. The specimen was then gently blotted.

\section{Imaging}


Imaging was performed using the previously described flexible probe 3D-printed in vivo fluorescence microscope ${ }^{11}$ (Fig. 1). A flexible fiber bundle (field of view, $790 \mu \mathrm{m}$ ) composed of 30,000 imaging elements (pixels) was used for imaging (FIGH-30-850N, Fujikura, Tokyo, Japan). Light-emitting diode (LED) excitation light specific to the excitation wavelength of acridine orange $(\sim 450 \mathrm{~nm})$ is delivered to the tissues through the imaging fiber bundle. Emitted fluorescence $(\sim 550 \mathrm{~nm})$ is then carried back through the fiber bundle to an $\times 20$ objective lens and filters to be captured using a consumer-grade digital camera. Imaging an area of tissue was performed by manually scanning the end of the flexible fiber against the tissue while sequential images or videos were recorded (Fig. 1). A real-time video feed was projected to a screen during the imaging session, which allowed for guidance during imaging.

\section{Image Processing and Analysis}

To increase imaging resolution and field of imaging, sequential images or frames from a recorded video sequence were stacked and aligned on the basis of the scale-invariant feature transform (SIFT) ${ }^{12}$ implemented through FIJI (FIJI is just ImageJ) ${ }^{13}$ (Fig. 2). Frames or images with motion blur were excluded before alignment. Maximum signal projection was then performed to reduce noise and reduce honeycomb artifact superimposed on the images by the fiber bundle. Finally, contrast is enhanced using contrast-limited adaptive histogram equalization implemented through FIJI. ${ }^{14}$ The resulting images also represented a mosaic over the area scanned, thereby effectively increasing the imaged field size. Approximated pseudo-hematoxylin and eosin $(\mathrm{H} \& \mathrm{E})$ colorization of the fluorescence images was performed to enhance the detection of features (Fig. 2). Colorization was performed after gray-scale fluorescent images were first corrected for illumination. Bright fluorescence was considered to represent the nuclear (hematoxylin) channel, and inverted images were considered to simulate eosin staining. A method similar to that described by Gareau ${ }^{15}$ was then performed in which H\&E channels are colored via a look-up table and then combined. After imaging, the specimens were placed in formalin and processed using routine histologic processing. The Clinical and Laboratory Images in Publications (CLIP) guidelines were used in the reporting of images in this study. ${ }^{16}$ 


\section{Results}

All ex vivo specimens demonstrated good nuclear fluorescence with acridine orange (representative images are shown in Figs. 3-7). Nuclear size, pleomorphism, and cellularity were evident in all specimens. 3D structures, such as 3D fascicles in meningiomas and cord-like vessels, were identifiable. Larger vessels in cut sections appeared as dark, branching, hollow structures traversing the tissue. The nuclei of vascular endothelial cells were seen arranged in a cylindrical fashion around the vascular lumens.

Although only tumorous tissue was collected for the purposes of this study, normal cerebellar tissue was seen in 1 specimen (specimen 18). Cerebellar gray matter showed small, moderately bright, equidistant nuclei in flat sheets with a diffuse low-brightness background representing the neuropil. An abrupt distinction was seen at the interface of the molecular layer with the highly cellular granular layer (Fig. 3).

The resolution was maximally enhanced in the center of the mosaicked image where most frames overlapped. Sequences with higher overlap between frames resulted in higher-resolution images. Pseudo-coloring resulted in images that visually resembled H\&E stains (Figs. 2C, 7B, and 7F). Pseudo-coloring transformed the bright staining nuclei to hematoxylin-like blue and the moderately stained cytoplasm surrounding nuclei to eosin-like pink. Spaces between cells appeared white, but red blood cells were not visible after pseudo-coloring.

\section{Meningioma}

Fluorescence imaging demonstrated a 3D arrangement of meningothelial cells in fascicles and whorls (Fig. 4A, B). The nuclei showed characteristic streaming in most of the meningiomas imaged. Image mosaicking and stitching increased the image area, thereby enhancing architectural assessment of the tumors. Rare intranuclear inclusions were detected with the aid of enhanced resolution after processing. Psammoma bodies appeared round to oval with a smooth, bright border and a dark center (Fig 4C). An example of a meningothelial meningioma displaying typical meningothelial whorls and sparse psammoma bodies is shown in Fig 4. In grade 1 meningiomas, the nuclei were equidistant, small, and showed no pleomorphism. Highergrade meningiomas showed little or no streaming and increased nuclear size; however, mitoses 
were not easily identified. Different architectural patterns, such as a microcystic pattern, were identifiable. In microcystic meningiomas, dark, round, empty spaces were seen between the chords of meningothelial cells.

\section{Oligodendroglioma}

Oligodendroglioma exhibited striking nuclear monotony and regularity. The nuclei were circular and evenly distributed. The background showed various-intensity staining; however, some cells demonstrated darker cell boundaries and cytoplasm (Fig. 5B). Compared with H\&E, the nuclear density appeared increased with this imaging method because several en face planes of tissues were imaged at once. The "chicken-wire" arrangement of vessels was not easily identified; however, dark "cracks" appeared to separate the tissue into irregular lobules (Fig. 5A). This cracking may be an artifact of tissue surface fragmentation during imaging.

\section{Pituitary Adenoma}

The ex vivo sample of pituitary adenoma imaged was predominantly composed of blood clot and fibrin (Fig. 6B). These appeared as amorphous structures with scattered small, round, black structures resembling red blood cells. Scattered nests of adenomatous tissue were visible throughout the sample. These cells showed regular round nuclei, and the cytoplasm appeared dark. Further distinction of acidophilic or basophilic cytoplasm was not feasible.

\section{Glioblastoma}

Glioblastomas exhibited marked nuclear pleomorphism. The nuclei were arranged haphazardly with abrupt, large, bright nuclei. Mitotic figures were best detected after pseudo-coloring, demonstrating the utility of this technique in aiding diagnosis (Fig. 7B). Occasionally, pleomorphic multinucleated cells were also seen (Fig. 7C). Increased vascularity was seen during scanning of the fiber probe. Small blood vessels appeared as 3D cords that rolled under the probe during manual scanning (Fig. 7D). Larger vessels that were cut in sections appeared as curvilinear dark branching spaces traversing the tissue. The endothelium of these vessels appeared thickened. Extravasated red blood cells were not easily identified; however, areas of necrosis appeared dark with nonspecific speckled fluorescence surrounded by nuclear pseudopalisading (Fig. 7F). 


\section{Discussion}

Fluorescence-guided tumor resection can increase the extent of resection and potentially improve patient progression-free and overall survival. ${ }^{17,18}$ The use of in vivo microscopy may also further improve outcome and/or patient survival. ${ }^{19}$ Multiple in vivo diagnostic imaging modalities have been studied for the use in the nervous system. Fluorescence microscopy is one such technology which shows promise for accurate diagnosis of tumors in vivo and ex vivo. ${ }^{20,21}$

The incorporation of flexible probes in in vivo microscopes enhances their utility. ${ }^{22-25}$ The flexible probe can be placed to image tissues that would otherwise be challenging to reach with a rigid or miniaturized microscope. ${ }^{26}$ The majority of studies using fluorescence, whether ex vivo or in vivo, have relied on confocal microscopy. ${ }^{19,27-29}$ Non-confocal (epifluorescence) in vivo microscopic techniques have also been shown to provide adequate imaging using simpler setups. ${ }^{30-32}$ One major drawback of using flexible probes for imaging is that images are severely pixelated (Fig. 2A). This pixilation is due to the fact that flexible imaging fiber bundles are composed of thousands of smaller fibers each acting as a pixel, resulting in a pixilated image with a honeycomb artifact (Fig. 2a). ${ }^{33,34}$

In this study, we demonstrate the enhancement of the imaging capability of flexible fiber-based imaging. Taking advantage of the high rate of acquisition of non-confocal fluorescence microscopes, stacking of multiple adjacent frames is possible to enhance resolution and increase field of view (Fig. 2). Lee et al. demonstrated the possibility of eliminating the fiber-bundle honeycomb artifact in optical coherence tomography images by superimposing four slightly offset images. ${ }^{35}$ We demonstrate a similar concept applied to fiber-bundle fluorescence imaging. Resolution enhancement was clearly demonstrated as otherwise invisible subpixel features such as mitotic figures were identifiable after processing. This processing may be performed in real time, allowing high-resolution imaging over large areas of tissue during neurosurgical procedures. In addition to resolution enhancement, this method automatically performs mosaicking, producing a large field of view that further enhances the diagnostic capability of fiber bundle imaging. ${ }^{36}$ Finally, we have demonstrated the feasibility of pseudo-coloring a single 
163

164

165

166

167

168

169

170

171

172

173

174

175

177

contrast agent to produce images that resemble H\&E-stained tissues (Figs. 2C, 7B, and 7F). Pseudo-coloring brings this in vivo microscopy technique one step closer to clinical adoptability and utility.

In vivo images generated using this system will vary according to the contrast agent used. The use of injected fluorescein is likely to result in images based on vascular contrast and vascular integrity. Dynamic changes such as increased fluorescein extravasation in malignant tumors may therefore be seen. ${ }^{37-39}$ In vivo imaging with acridine orange will provide nuclear contrast, improving the diagnostic ability compared to fluorescein. Acridine orange and other nuclear contrast agents such as acriflavine and proflavine, have been used as in vivo nuclear contrast agents for microscopy. ${ }^{40-44}$ The use of nuclear contrast agents in vivo for neuropathologic assessment may be of importance because nuclear assessment is critical for the diagnosis of many neurologic tumors. Acridine orange has also been recently demonstrated as a potential agent for neuro-oncologic photodynamic therapy. ${ }^{45}$ Its utility may therefore serve as both a therapeutic and an in vivo nuclear contrast agent.

\section{Conclusion}

We have demonstrated the ex vivo utility of an imaging fiberoptic based fluorescence microscope for obtaining high-resolution microscopic images comparable to those of standard histology. Implementation of this imaging technique in vivo may permit microscopic assessment of residual tumor cells at surgical margins and potentially improve surgical outcomes for patients with benign and malignant brain tumors.

\section{Funding Support}

This research did not receive any specific grant from funding agencies in the public, commercial, or not-for-profit sectors.

\section{References}

1. Salcman M. The value of cytoreductive surgery. Clin Neurosurg. 1994;41:464-488. 
189

190

191

192

193

194

195

196

197

198

199

200

201

202

203

204

205

206

207

208

209

210

211

212

213

214

215

216

217

218

219

220

221

222

223

224

225

226

227

228

229

230

231

2. Scott JN, Rewcastle NB, Brasher PM, et al. Long-term glioblastoma multiforme survivors: a population-based study. Can J Neurol Sci. 1998;25(3):197-201.

3. Sanai N, Polley M-Y, McDermott MW, Parsa AT, Berger MS. An extent of resection threshold for newly diagnosed glioblastomas. J Neurosurg. 2011;115(1):3-8.

4. Hardesty DA, Sanai N. The value of glioma extent of resection in the modern neurosurgical era. Front Neurol. 2012;3:140.

5. Sanai N, Berger MS. Glioma extent of resection and its impact on patient outcome. Neurosurgery. 2008;62(4):753-764; discussion 264-756.

6. Babu R, Adamson DC. Fluorescence-guided malignant glioma resections. Curr Drug Discov Technol. 2012;9(4):256-267.

7. Zhao S, Wu J, Wang C, et al. Intraoperative fluorescence-guided resection of high-grade malignant gliomas using 5-aminolevulinic acid-induced porphyrins: a systematic review and meta-analysis of prospective studies. PloS One. 2013;8(5):e63682.

8. Nanda A, Bir SC, Maiti TK, Konar SK, Missios S, Guthikonda B. Relevance of Simpson grading system and recurrence-free survival after surgery for World Health Organization Grade I meningioma. J Neurosurg. 2017;126(1):201-211.

9. Akçakaya MO, Göker B, Kasımcan MÖ, Hamamcıoğlu MK, Kırış T. Use of sodium fluorescein in meningioma surgery performed under the YELLOW-560 nm surgical microscope filter: feasibility and preliminary results. World Neurosurg. 2017;107:966973.

10. Osman H, Georges J, Elsahy D, Hattab EM, Yocom S, Cohen-Gadol AA. In Vivo Microscopy in Neurosurgical Oncology. World Neurosurg. 2018;115:110-127.

11. Osman H. 3D Printable In-Situ Fluorescent Microscope. Microsc Microanal. 2015;21(S3):623-624.

12. Lowe DG. Distinctive image features from scale-invariant keypoints. Int J Comput Vis. 2004;60(2):91-110.

13. Schindelin J, Arganda-Carreras I, Frise E, et al. Fiji: an open-source platform for biological-image analysis. Nat Methods. 2012;9(7):676.

14. Pizer SM, Amburn EP, Austin JD, et al. Adaptive histogram equalization and its variations. Computer vision, graphics, and image processing. 1987;39(3):355-368.

15. Gareau DS. Feasibility of digitally stained multimodal confocal mosaics to simulate histopathology. J Biomed Opt. 2009;14(3):034050.

16. Lang TA, Talerico C, Siontis GC. Documenting clinical and laboratory images in publications: the CLIP principles. Chest. 2012;141(6):1626-1632.

17. Stummer W, Pichlmeier U, Meinel T, Wiestler OD, Zanella F, Reulen HJ. Fluorescenceguided surgery with 5-aminolevulinic acid for resection of malignant glioma: a randomised controlled multicentre phase III trial. Lancet Oncol. 2006;7(5):392-401.

18. McGirt MJ, Chaichana KL, Gathinji M, et al. Independent association of extent of resection with survival in patients with malignant brain astrocytoma. $J$ Neurosurg. 2009;110(1):156-162.

19. Sanai N, Eschbacher J, Hattendorf G, et al. Intraoperative confocal microscopy for brain tumors: a feasibility analysis in humans. Oper Neurosurg. 2011;68(suppl_2):ons282ons290. 
20. Martirosyan NL, Georges J, Eschbacher JM, et al. Confocal scanning microscopy provides rapid, detailed intraoperative histological assessment of brain neoplasms: Experience with 106 cases. Clinical Neurol Neurosurg. 2018;169:21-28.

21. Martirosyan NL, Eschbacher JM, Kalani MY, et al. Prospective evaluation of the utility of intraoperative confocal laser endomicroscopy in patients with brain neoplasms using fluorescein sodium: experience with 74 cases. Neurosurg Focus. 2016;40(3):E11.

22. Wang KK, Carr-Locke DL, Singh SK, et al. Use of probe-based confocal laser endomicroscopy ( $\mathrm{pCLE}$ ) in gastrointestinal applications. A consensus report based on clinical evidence. United European Gastroenterol J. 2015;3(3):230-254.

23. Wallace $\mathrm{M}$, Lauwers $\mathrm{GY}, \mathrm{Chen} \mathrm{Y}$, et al. Miami classification for probe-based confocal laser endomicroscopy. Endoscopy. 2011;43(10):882-891.

24. Thiberville L, Salaun M, Lachkar S, et al. Confocal fluorescence endomicroscopy of the human airways. Proceedings of the American Thoracic Society. 2009;6(5):444-449.

25. Nakai $\mathrm{Y}$, Isayama $\mathrm{H}$, Shinoura $\mathrm{S}$, et al. Confocal laser endomicroscopy in gastrointestinal and pancreatobiliary diseases. Dig Endosc. 2014;26 Suppl 1:86-94.

26. Hansell CW. Picture transmission. In: US Patent 1,751,584; 1930.

27. Pavlov V, Meyronet D, Meyer-Bisch V, et al. Intraoperative probe-based confocal laser endomicroscopy in surgery and stereotactic biopsy of low-grade and high-grade gliomas: a feasibility study in humans. Neurosurgery. 2016;79(4):604-612.

28. Martirosyan NL, Georges J, Eschbacher JM, et al. Potential application of a handheld confocal endomicroscope imaging system using a variety of fluorophores in experimental gliomas and normal brain. Neurosurg Focus. 2014;36(2):E16.

29. Martirosyan NL, Eschbacher JM, Kalani MYS, et al. Prospective evaluation of the utility of intraoperative confocal laser endomicroscopy in patients with brain neoplasms using fluorescein sodium: experience with 74 cases. Neurosurg Focus. 2016;40(3):E11.

30. Pierce M, Yu D, Richards-Kortum R. High-resolution fiber-optic microendoscopy for in situ cellular imaging. J Vis Exp. 2011(47).

31. Shin D, Pierce MC, Gillenwater AM, Williams MD, Richards-Kortum RR. A fiber-optic fluorescence microscope using a consumer-grade digital camera for in vivo cellular imaging. PLoS One. 2010;5(6):e11218.

32. Pierce MC, Guan Y, Quinn MK, et al. A pilot study of low-cost, high-resolution microendoscopy as a tool for identifying women with cervical precancer. Cancer Prev Res. 2012:canprevres. 0221.2012.

33. Han J-H, Lee J, Kang JU. Pixelation effect removal from fiber bundle probe based optical coherence tomography imaging. Opt Express. 2010;18(7):7427-7439.

34. Bazinet Jr Wilfred P, Hicks Jr John W. Fiber optical devices. In: Google Patents; 1961.

35. Lee $\mathrm{C}-\mathrm{Y}$, Han J-H. Elimination of honeycomb patterns in fiber bundle imaging by a superimposition method. Opt Lett. 2013;38(12):2023-2025.

36. Bedard N, Quang T, Schmeler K, Richards-Kortum R, Tkaczyk TS. Real-time video mosaicing with a high-resolution microendoscope. Biomed Opt Express. 2012;3(10):2428-2435.

37. Foersch S, Heimann A, Ayyad A, et al. Confocal laser endomicroscopy for diagnosis and histomorphologic imaging of brain tumors in vivo. PloS One. 2012;7(7):e41760. 
38. Charalampaki P, Javed M, Daali S, Heiroth HJ, Igressa A, Weber F. Confocal Laser Endomicroscopy for Real-time Histomorphological Diagnosis: Our Clinical Experience With 150 Brain and Spinal Tumor Cases. Neurosurgery. 2015;62 Suppl 1:171-176.

39. Pavlov V, Meyronet D, Meyer-Bisch V, et al. Intraoperative Probe-Based Confocal Laser Endomicroscopy in Surgery and Stereotactic Biopsy of Low-Grade and High-Grade Gliomas: A Feasibility Study in Humans. Neurosurgery. 2016;79(4):604-612.

40. Kiesslich R, Burg J, Vieth M, et al. Confocal laser endoscopy for diagnosing intraepithelial neoplasias and colorectal cancer in vivo. Gastroenterology. 2004;127(3):706-713.

41. Polglase AL, McLaren WJ, Skinner SA, Kiesslich R, Neurath MF, Delaney PM. A fluorescence confocal endomicroscope for in vivo microscopy of the upper-and the lower-GI tract. Gastrointest Endosc. 2005;62(5):686-695.

42. Pierce MC, Schwarz RA, Bhattar VS, et al. Accuracy of in vivo multi-modal optical imaging for detection of oral neoplasia. Cancer Prev Res. 2012:canprevres. 0555.2011.

43. Tanbakuchi AA, Udovich JA, Rouse AR, Hatch KD, Gmitro AF. In vivo imaging of ovarian tissue using a novel confocal microlaparoscope. Am J Obstet Gynecol. 2010;202(1):90. e91-90. e99.

44. Satonaka $\mathrm{H}$, Kusuzaki $\mathrm{K}$, Matsubara $\mathrm{T}$, et al. In vivo anti-tumor activity of photodynamic therapy with intravenous administration of acridine orange, followed by illumination with high-power flash wave light in a mouse osteosarcoma model. Oncology Lett. 2010;1(1):69-72.

45. Osman H, Elsahy D, Saadatzadeh MR, et al. Acridine Orange as a Novel Photosensitizer for Photodynamic Therapy in Glioblastoma. World Neurosurg. 2018;114:e1310-e1315.

Fig. 1. Image acquisition system. A: The tip of the fiber is placed against the tissue to be imaged. The fiber is manually scanned against the tissue. B: Images are transmitted through the fiber to the 3D-printed microscope. C: Images are displayed in real time on a connected monitor.

Fig. 2. Image-processing procedure. A: Several frames from a video sequence aligned using the scale-invariant feature transform (SIFT) algorithm. ${ }^{16}$ Inset is an enlarged section (corresponding to boxed area) showing significant fiber bundle pixilation (honeycomb artifact). B: Maximum intensity projection (superimposition) and contrast enhancement forming a larger-area mosaic with enhanced resolution. Inset is an enlargement corresponding to boxed areas showing increased resolution. C: H\&E pseudo-coloring, Inset is an enlargement that corresponds to boxed area.

Fig. 3. A: Histologic section of normal cerebellum showing normal molecular and granular cell layers. B: Corresponding processed fiber probe images demonstrating contrast between layers. 
Molecular layer shows small equidistant nuclei embedded in a gray background. The highly cellular granular layer is composed of densely packed small, round, and oval nuclei.

Fig. 4. Processed fiber probe images of a meningothelial meningioma demonstrating characteristic whorls (A), fascicles of cells with streaming nuclei (B), and psammoma bodies (C). D: Corresponding H\&E-stained tissue from the same sample showing classic features of meningioma, including fasciculation and whorling.

Fig. 5. Oligodendroglioma. A: Processed fiber probe images showing sheets of monomorphic, equally spaced nuclei. B: Magnification shows round, bright nuclei, some surrounded with a variable background. C and D: H\&E-stained tissue from the same sample showing characteristic findings of oligodendroglioma, including round monomorphic nuclei, and chicken-wire arrangement of capillaries.

Fig. 6. Pituitary adenoma. A: Processed fiber probe images showing nests of cells with bright, round nuclei surrounded by amorphous fibrillary material containing round, dark bodies resembling red blood cells. B: H\&E-stained tissue from the same sample showing predominantly blood and fibrin with scattered nests of pituitary adenoma cells.

Fig. 7. Glioblastoma. A: Processed fiber probe images showing striking disarrangement and pleomorphism consistent with a glioblastoma. B: Pseudo-colored image corresponding to the image in panel a showing features resembling those of a standard H\&E stain, including the presence of mitoses (see inset image) and binucleated pleomorphic cells. C: H\&E-stained tissue from the same sample showing similar features of striking pleomorphism, mitoses, and binucleated cells. D: Increased vessels seen as branching serpentine cords. E: An area of focal necrosis seen as lack of nuclei, a variably staining necrotic core (arrowhead), and pseudopalisading of nuclei. F: Pseudo-colored image corresponding to the image in panel E showing focal necrosis (arrowhead) and pseudo-palisading. 
Table 1. Ex vivo specimens examined

\begin{tabular}{ll}
\hline Specimen No. & Diagnosis \\
\hline 1 & Meningioma, meningothelial, WHO grade I \\
2 & Anaplastic meningioma, WHO grade III \\
3 & Meningioma, microcystic, WHO grade I \\
4 & Meningioma, WHO grade I \\
5 & Meningioma, transitional, WHO grade I \\
6 & Meningioma, transitional, WHO grade I \\
7 & Meningioma, transitional, WHO grade I \\
8 & Meningioma, WHO grade I \\
9 & Meningioma, WHO grade I \\
10 & Meningioma, meningothelial, WHO grade I \\
11 & Meningioma, WHO grade I \\
12 & Glioblastoma, WHO grade IV \\
13 & Atypical meningioma, WHO grade II \\
14 & Glioblastoma, WHO grade IV \\
15 & Oligodendroglioma, WHO grade II \\
16 & Pituitary adenoma \\
17 & Meningioma, WHO grade I \\
18 & Metastatic malignancy (tissue imaged was \\
& composed of normal cerebellum) \\
\hline
\end{tabular}

Abbreviation: WHO, World Health Organization. 


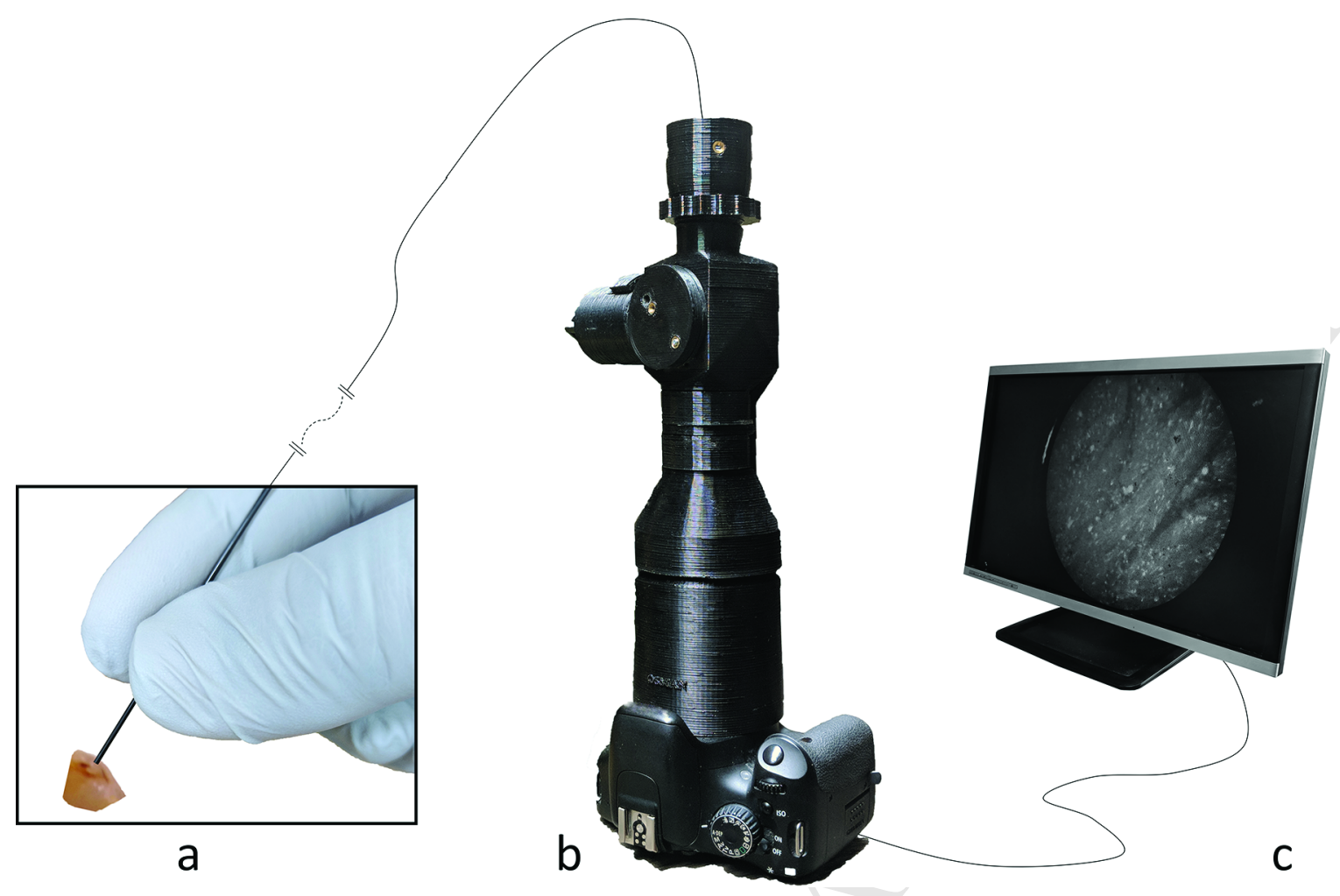



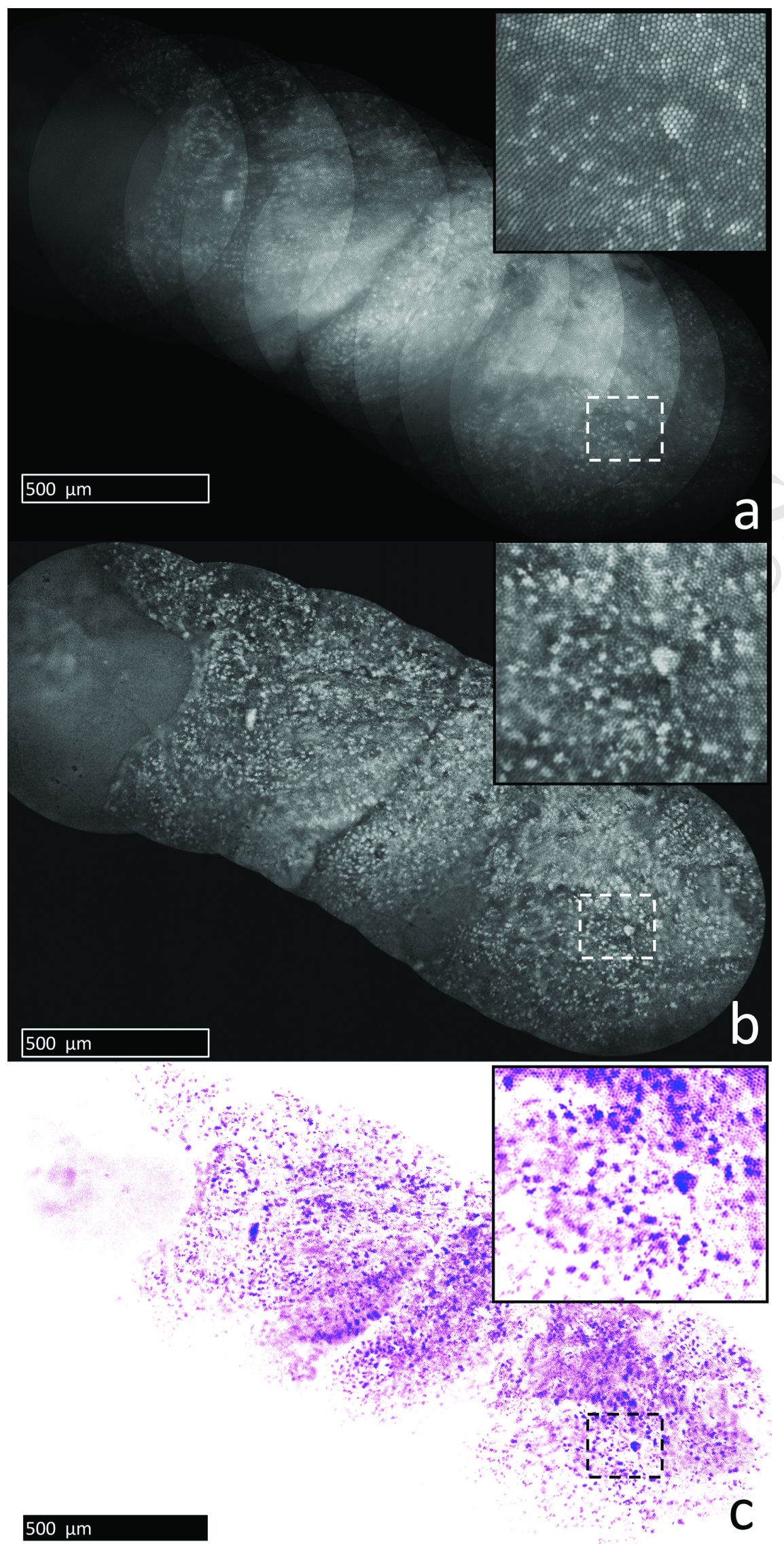


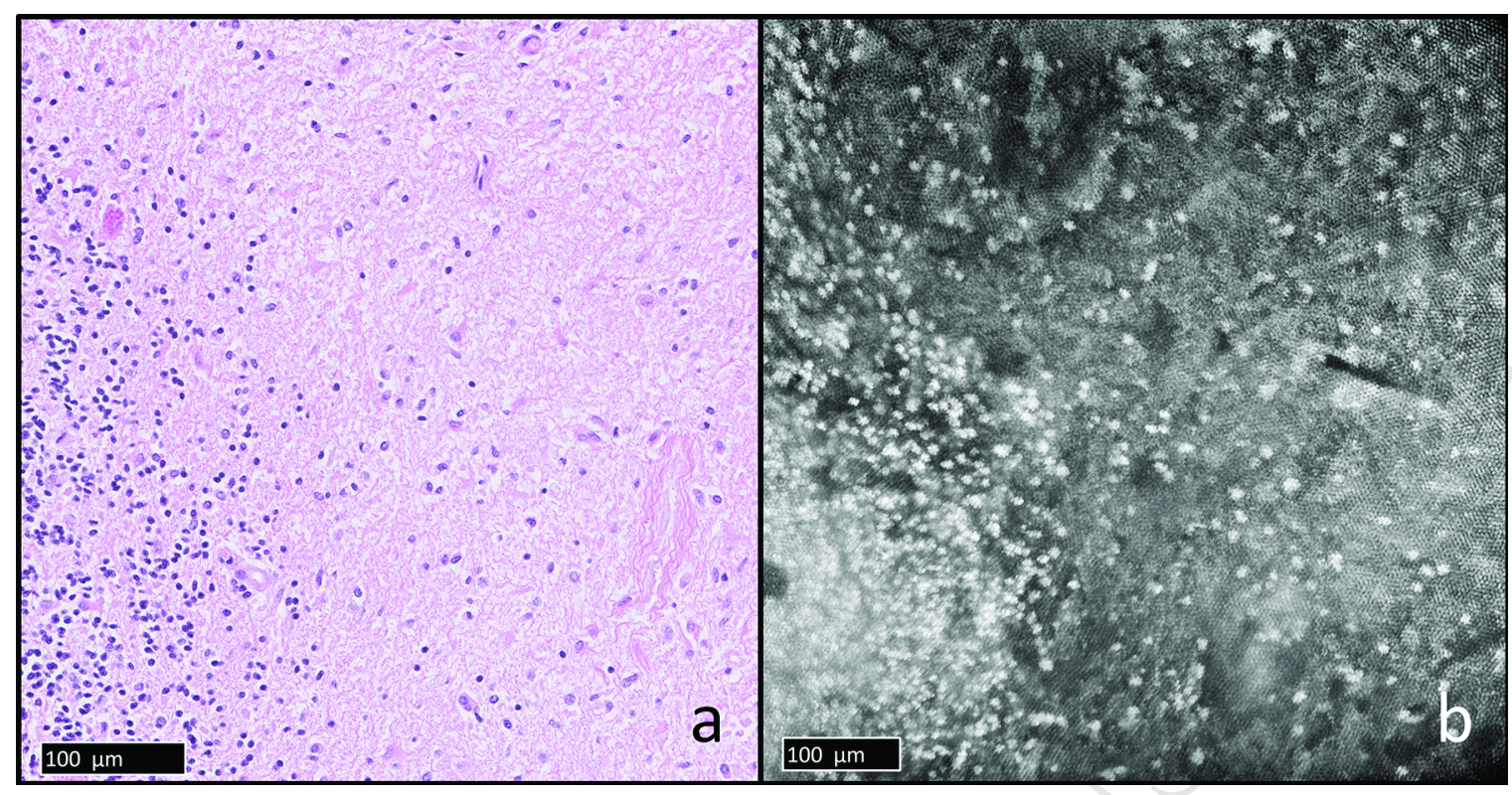




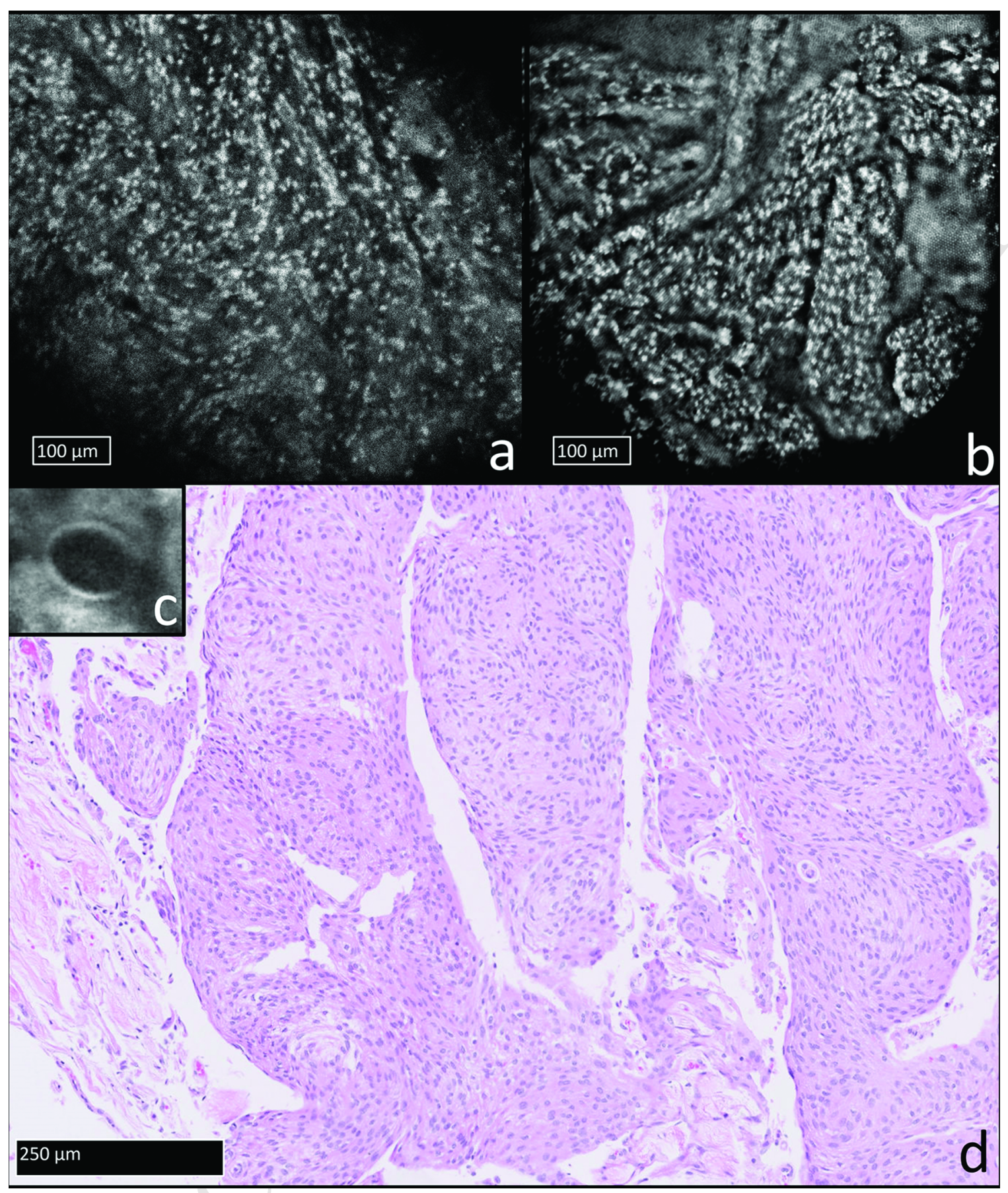









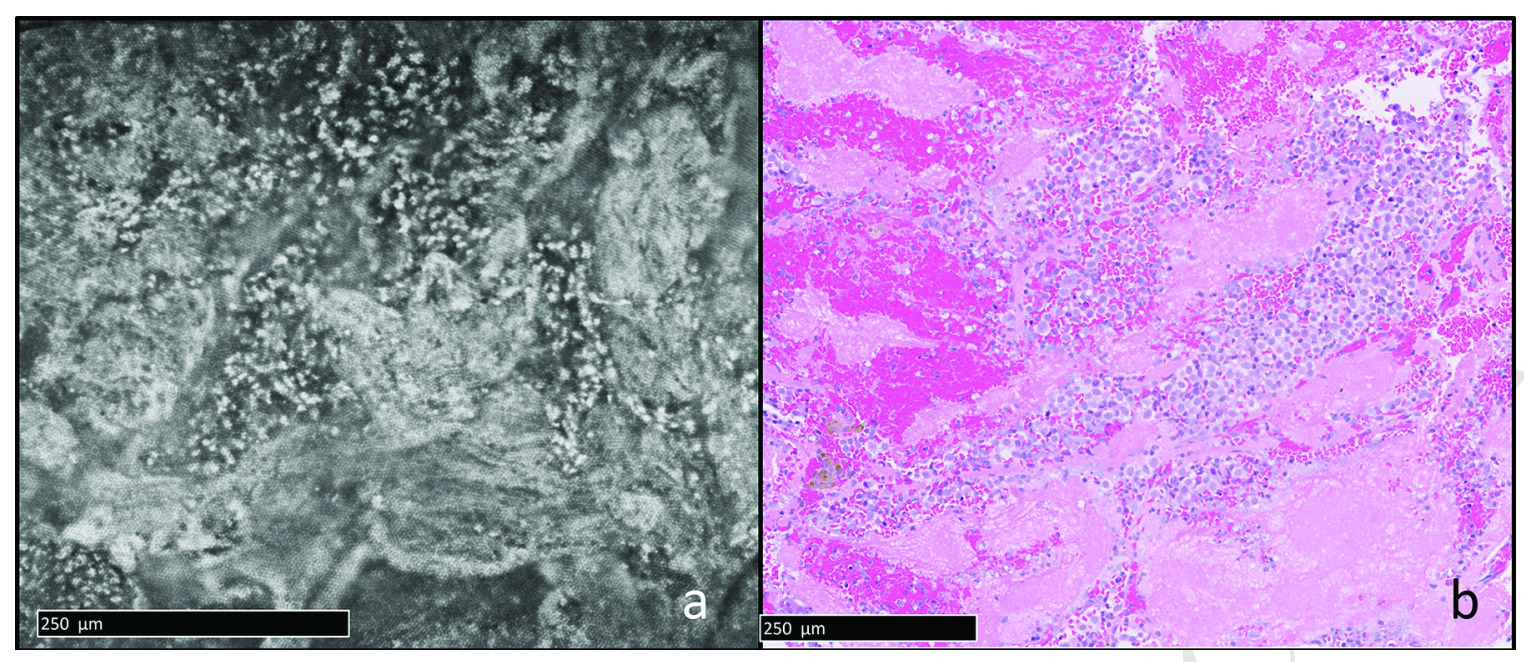




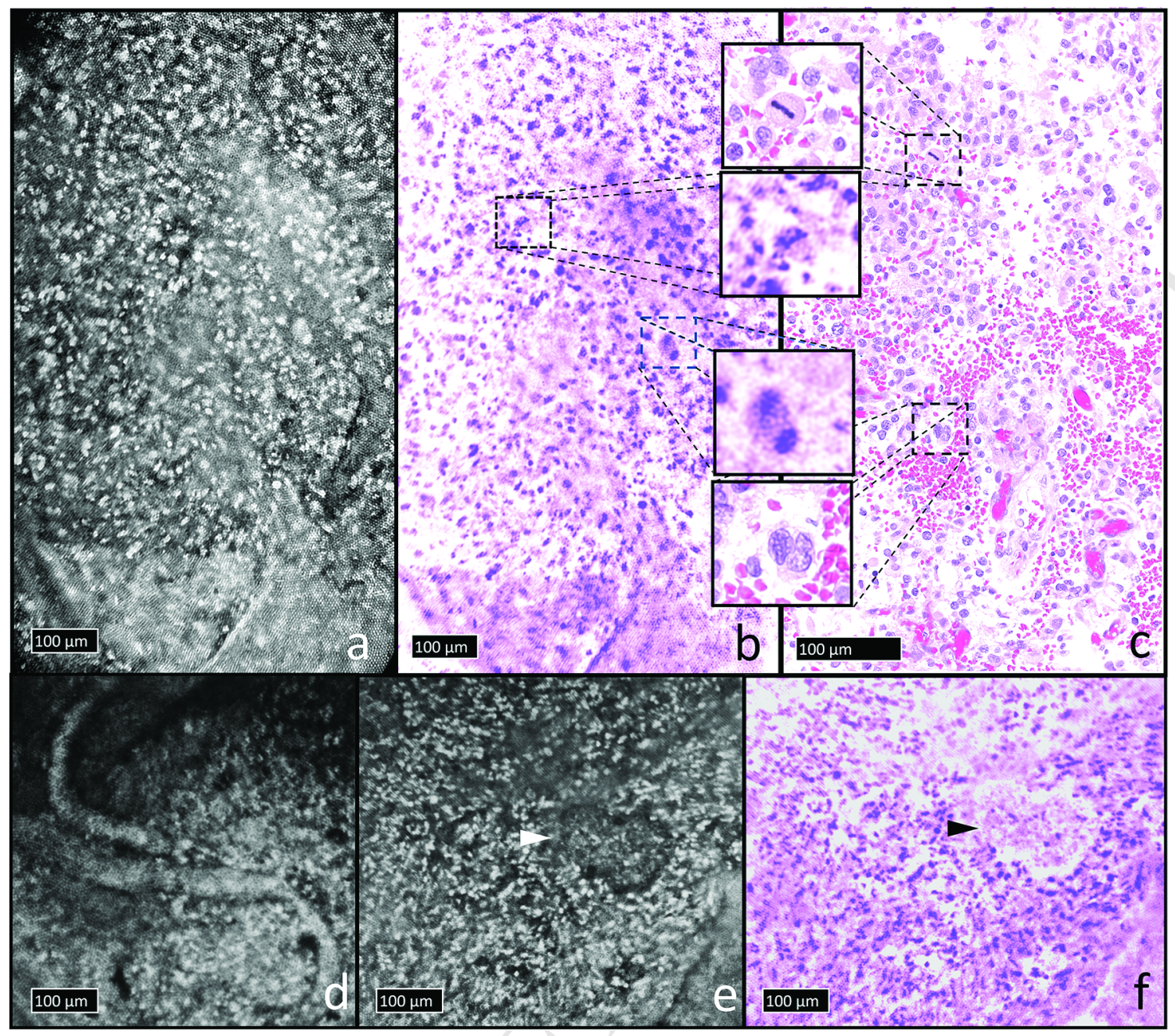




\section{Abbreviations}

CLIP, Clinical and Laboratory Images in Publications

FIJI, FIJI is just ImageJ

H\&E, hematoxylin and eosin

LED, light-emitting diode

SIFT, scale-invariant feature transform

WHO, World Health Organization 